\title{
La adquisición de vocabulario mediante proyectos: una experiencia docente ${ }^{1}$
}

\section{(Using Projects for the Acquisition of Vocabulary: A Teaching Experience)}

\author{
Asunción Martínez-Arbelaiz ${ }^{2}$ \\ University Studies Abroad Consortium (USAC), San Sebastián, España \\ Natalia Errazquin ${ }^{3}$ \\ USAC, San Sebastián, España \\ MariMar Olano ${ }^{4}$ \\ USAC, San Sebastián, España
}

\begin{abstract}
resumen
Adoptando una metodología por proyectos, realizamos una experiencia docente con un grupo de seis alumnos de español como segunda lengua en situación de inmersión. Seleccionamos sesenta y cuatro piezas léxicas, que el grupo dijo que eran nuevas para ellos, todas relacionadas con la historia del País Vasco. Los alumnos realizaron tareas de producción y de centrarse en la forma que incluían dicho vocabulario, las cuales se describen aquí. Los test administrados al terminar el proyecto dieron evidencia de que el léxico había sido fijado en la memoria. Siete semanas después los resultados se mantuvieron. Concluimos, por lo tanto, que el trabajo por proyectos
\end{abstract}

1 Elaborado con base en la ponencia presentada por las autoras en el IV Congreso Internacional de Lingüística Aplicada, llevado a cabo en mayo de 2013, en el Campus Omar Dengo, de la Universidad Nacional de Costa Rica. Recibido: 19 de agosto de 2012; aceptado: 13 de enero de 2013.

2 Correo electrónico: asuncion@usac.unr.edux

3 Correo electrónico: nataliaerrazkin@yahoo.com

4 Correo electrónico: mmoo.web@ gmail.com 
repercute positivamente en la adquisición de nuevo léxico, no solo a corto plazo sino que el efecto es duradero.

\section{abstract}

Adopting a project-based methodology, we carried out a teaching experience with a group of six students of Spanish as a second language in an immersion setting. We selected sixty-four lexical items that the students reported to be new to them, related to the history of the Basque Country. The students completed several production and focus-on-form tasks (described here), which included that vocabulary. The tests administered after finishing the project provided evidence that the lexicon had been fixed in the memory. Seven weeks later the same results were obtained. We conclude, therefore, that project-based work has a positive impact on the acquisition of new vocabulary, not only for short-term memory but also for long-term memory.

Palabras clave: adquisición léxica, memoria a corto y largo plazo, aprendizaje por proyectos, foco en las formas

Keywords: lexical acquisition, short/long-term memory, project-based learning, focus on forms

\section{Introducción}

Los profesores, tanto de primera como de segundas lenguas, a menudo pensamos que un alumno sabe una palabra cuando es capaz de comprender su significado en una situación comunicativa dada. De ahí que la adquisición de vocabulario, tanto en la investigación como en la práctica docente, suele aparecer ligada a la lectura extensiva por la creencia generalizada de que cuanto más lea un alumno, más vocabulario aprenderá 5 . Aunque la propuesta que afirma que la lectura extensiva favorece la adquisición del léxico no está exenta de polémica, sigue siendo una práctica desarrollada asignar lecturas en la lengua meta para mejorar el vocabulario de los alumnos. Bien es cierto que los profesores de segundas lenguas nos mostramos

5 Stephen Krashen, «We Acquire Vocabulary and Spelling by Reading: Additional Evidence for the Input Hypothesis», The Modern Language Journal, 73 (1989): 440-464. 
satisfechos si el alumno puede entender un texto dado, asumiendo que entiende las piezas léxicas de las que consta.

Sin embargo, autores como Ellis y Beaton sostienen que el conocimiento de una palabra implica más que el puro desciframiento de su significado ${ }^{6}$. En concreto, proponen que saber una palabra implica un conjunto o un haz de rasgos, tales como conocer su ortografía, su representación fonológica, la categoría gramatical a la que pertenece, sus rasgos morfológicos o sintácticos, su significado básico, así como los secundarios que pueda tener, entre otros aspectos. Más recientemente, se ha añadido a esta lista el conocimiento de colocaciones, o de las solidaridades léxicas que se establecen entre palabras ${ }^{7}$. En cualquier caso, la adquisición léxica es un proceso largo y complejo que no puede reducirse a la mera comprensión de los ítems léxicos en cuestión.

Desde un punto de vista pedagógico y de acción en la clase, un factor que incide en la adquisición y que más ha recalcado la investigación, es el número de veces que el alumno tiene que acceder a una pieza léxica, sea de forma productiva o receptiva. Como resume Folse, « $[\mathrm{t}] \mathrm{hus}$, the most important point in teaching and learning vocabulary is the number of times the learner has to retrieve the word, a factor which the teacher can influence through classroom activities» ${ }^{8}$.

Un segundo factor que parece influir en si el aprendiz adquiere o no un ítem léxico es el tipo de actividad que lleva a cabo. Cognitivamente no es lo mismo procesar un ítem durante la lectura de un texto que tener que traer a la memoria uno léxico cuando haya que completar una tarea escrita, como por ejemplo, al redactar una descripción o una narración. Laufer y Rozovski-Roitblat ${ }^{9}$ distinguen entre el

6 N. Ellis y A. Beaton, «Psycholinguistic Determinants of Foreign Language Vocabulary», Language Learning, 43 (1993): 559-617.

7 Marta Higueras, Las colocaciones y la enseñanza de ELE (Madrid: Arco Libros, 2006).

8 Keith Folse, «The Underestimated Importance of Vocabulary in the Foreign Language Classroom», Clear News, 8.2 (2004): 1-6.

9 B. Laufer y B. Rozovski-Roitblat, «Incidental Vocabulary Acquisition: The Effects of Task Type, Word Occurrence and their Combination», Language Teaching Research 15.4 (2011): 391-411. 
foco en la forma y el foco en las formas en la adquisición de léxico, haciendo eco de la famosa y conocida distinción de Long ${ }^{10}$. Aunque Long originalmente ideó esta distinción teniendo en cuenta los procesos de adquisición de puntos gramaticales, Laufer la extendió a la de vocabulario ${ }^{11}$. Resumiendo: el foco, en la forma, sería simplemente cuando el estudiante lee y busca las palabras que va necesitando en un diccionario. No se asocia con ninguna actividad específica que haga que el alumno preste atención a unas palabras u otras. De esta manera, se trata de un aprendizaje puramente incidental. El foco en las formas, por el contrario, requiere que el profesor diseñe actividades para dirigir la atención del alumno hacia representaciones léxicas previamente seleccionadas. Una actividad prototípica de foco en las formas sería un ejercicio de relacionar columnas de palabras con su sinónimo o su equivalente en la primera lengua de los alumnos.

Los resultados del estudio de Laufer y Rozovski-Roitblat apuntan a la idea de que es recomendable que los estudiantes realicen actividades de foco en las formas, es decir, actividades que les haga fijarse en formas léxicas previamente seleccionadas para así recordarlas. Estas actividades pueden ser tanto de reconocimiento como de producción. Asimismo, en este estudio se concluye que el número de veces ideal que el aprendiz tiene que enfrentarse a una palabra para que la recuerde son unas cuatro.

\section{Adquisición de léxico en el contexto de un proyecto}

Se ha mostrado que el trabajo por proyectos (entendiendo el proyecto como una sucesión de tareas a partir de un tema cultural

10 Michael H. Long, «Focus on Form: A Design Feature in Language Teaching Methodology», Foreign Language Research in Cross-Cultural Perspective. Kees de Bot, Claire Kramsch y Ralph B. Ginsberg, Eds. (Amsterdam/Filadelfia, PA: John Benjamins 1991) 39-51.

11 Batia Laufer, «Focus on Form in Second Language Vocabulary Learning», Eurosla Yearbook, Vol. 5. Susan H. Foster-Cohen, María Pilar García-Mayo y Jasone Cenoz, Eds. Amsterdam/Filadelfia. PA: John Benjamins (2005): 223-50. 
relacionado con la realidad que rodea al alumno ${ }^{12}$ ) se revierte en la adquisición léxica. Así, cuando los estudiantes eran los responsables de buscar, definir y compartir el vocabulario con sus compañeros, se favorece que utilicen esta terminología de forma oral en sus presentaciones y de forma escrita en sus redacciones. Estos resultados se explican a partir de la hipótesis de la carga de participación (Involvement Load Hypothesis de Laufer y Hulstijn), puesto que esta es alta en las actividades que requieren la producción de ítems léxicos seleccionados por los propios alumnos. Esta hipótesis también incluye un componente motivacional, asociado con la necesidad de usar una palabra así como dos unidades cognitivas: la búsqueda tanto del significado de una palabra como del término que corresponde a un contenido dado, y la evaluación, entendida como la comparación con otros ítems léxicos o el significado de la palabra con otros significados que esta tenga ${ }^{13}$.

Por otro lado, De la Fuente muestra que el tipo de instrucción tiene un impacto en la adquisición de vocabulario. Esta investigadora compara una metodología basada en tareas con la presentación, práctica y producción de un léxico dado. Los alumnos recuerdan mejor el vocabulario estudiado por medio de tareas, efecto que se manifiesta a largo plazo, es decir una semana después. Sin embargo, no se observa diferencia entre ambos tipos de instrucción inmediatamente después de la práctica. Este estudio, por lo tanto, da evidencia en favor de la presentación de vocabulario nuevo en el marco de tareas que requieran de su manipulación directa. También muestra que cuando la producción del léxico no se requiere desde el diseño de la tarea, se ve que los aprendices optan por caminos más trillados, evitando el uso de léxico muy específico. Finalmente, señala que una explicación por parte del profesor sobre la corrección en cuanto a la

12 Soledad Fernández, «Introducción», Tareas y proyectos en clase. S. Fernández, Ed. (Madrid: Edinumen, 2001) 13-38.

13 B. Laufer y J. Hulstijn, «Incidental Vocabulary Acquisition in a Second Language: The Construct of Task-Induced Involvement», Applied Linguistics 22 (2001): 1-26. 
marcación de género y número del vocabulario, revierte en la correcta adquisición no solo del significado de la palabra, sino también en los aspectos morfosintácticos de esta ${ }^{14}$.

Resumiendo: es fundamental para la adquisición de vocabulario diseñar escrupulosamente contextos en los que los alumnos tengan que usar una determinada terminología, a la vez que el profesor incide en las características formales de dicho léxico, bien sea a través de explicaciones o de feedback, directo o indirecto. Parece necesario proporcionar numerosas ocasiones para su uso y respecto a esto, algunos autores precisan que la aparición o mejor, la producción de estas palabras cuatro o cinco veces, facilitan su retención.

\section{Metodología}

A continuación detallamos la propuesta didáctica que se llevó a cabo con un grupo de alumnos de lengua materna inglesa que estudiaban español en San Sebastián, España. La propuesta se dividió en varias fases, tanto dentro como fuera del aula. Los seis alumnos se dividieron en tres parejas y se les repartieron diferentes textos para realizar una pequeña tarea de investigación. Los temas estaban relacionados con el contexto de instrucción, el País Vasco, entendido en su sentido más amplio y cultural, y se concretaron en las siguientes actividades:

1. Presentación oral de un tema previamente fijado y selección de los ítems léxicos: 17 de octubre de 2011

2. Escritura en casa sobre el tema de la brujería: 19 de octubre de 2011

3. Ejercicios de foco en las formas: identificar imágenes; relacionar la palabra y su definición; a partir de la definición, recordar la palabra: 20 de octubre de 2011

14 M. José de la Fuente, «Classroom L2 Vocabulary Acquisition: Investigating the Role of Pedagogical Tasks and Form-Focused Instruction», Language Teaching Research X, 3 (2006): 263-295. 
4. Escritura en clase en el marco de un examen: 24 de octubre de 2011

5. Test de memoria a corto plazo: 26 de octubre de 2011

6. Visionado de la película Akelarre ${ }^{15}: 27$ de octubre de 2011

7. Test de memoria a largo plazo: 12 de diciembre de 2011

La secuencia de actividades de las que constaba el proyecto, la metodología integra la comprensión con la producción tanto oral como escrita de los ítems léxicos en cuestión. Las tareas de producción escrita se realizaron fuera y dentro del aula, eligiéndose temas relacionados para facilitar el reciclado del vocabulario en cuestión. Asimismo, las tareas de foco en las formas, fueron de diferentes tipos, incluyendo imágenes y definiciones, para evitar la monotonía y potenciar la variedad del tipo de actividades.

\section{Procedimiento}

\section{Participantes}

Los participantes fueron seis alumnos de habla inglesa y de nacionalidad estadounidense matriculados en un curso de español como lengua extranjera impartido en San Sebastián. Asistían a esta clase y contaban al menos con un nivel de B2 según el Marco Común de Referencia Europeo, por lo que el curso se podía enfocar en aquellos aspectos de perfeccionamiento o de expansión léxica. Se optó por una metodología por proyectos, aunque en ningún momento se desatendieron otros aspectos de índole más gramatical ${ }^{16}$.

\section{Contexto}

El contexto de aprendizaje de una segunda lengua es un aspecto que no ha recibido suficiente atención en los estudios de

15 Akelarre es una película dirigida por el bilbaíno Pedro Olea estrenada en 1984. En <http://es.paperblog. com/akelarre-435624>, se puede acceder a la sinopsis y algunas imágenes de dicha película.

16 Para una descripción completa de los cursos de nivel avanzado que ofrece USAC, se puede visitar <www.usac.unr.edu>. 
adquisición, hasta hace relativamente poco ${ }^{17}$. Se suelen oponer contextos de aprendizaje natural con los de aula, pero generalmente nos encontramos con panoramas híbridos o mixtos, en los que se aúna el aprendizaje formal típico de la clase con la necesidad de comunicación real que se experimenta fuera del aula ${ }^{18}$. Este estudio se centra en el contexto de inmersión o estudio de la lengua meta en el extranjero. En tal situación, los alumnos reciben instrucción formal en el aula pero, a diferencia de lo que ocurre en una clase de lengua extranjera, ellos tienen ocasiones de utilizar la lengua meta para fines concretos y prácticos fuera del aula.

Según Allen, muchos estudiantes que salen al extranjero a aprender una lengua, creen de forma más o menos consciente que la inmersión garantiza el aprendizaje de ella por medio de ósmosis y pocas veces se enfatiza lo suficiente el hecho de que el estudio de lenguas requiere esfuerzo continuado. Sin embargo, afirma que el contexto en sí no es lo relevante sino cómo se relaciona cada alumno en particular con este, es decir, el aprovechamiento de las oportunidades ilimitadas que el contexto proporciona ${ }^{19}$. Desde un punto de vista pedagógico, el contexto de inmersión da a la clase de lengua una dimensión diferente, puesto que se pueden diseñar actividades que incluyan interacción con personas del entorno o la observación crítica del paisaje lingüístico y cultural, algo difícil de conseguir en el aula de lengua extranjera ${ }^{20}$. Asimismo, la instrucción recibida en el aula suele ser de utilidad inmediata fuera, con lo que aumenta la motivación y el sentido que se les puede dar a las actividades de clase ${ }^{21}$.

17 Joseph Collentine, «Study Abroad Research: Finding, Implications, and Future Directions», The Handbook of Language Teaching. Michael H. Long y Catherine J. Doughty, Eds. (Oxford: WileyBlackwell, 2009) 218-233.

18 J. Cenoz, y J. Perales, «Las variables contextuales y el efecto de la instrucción en la adquisición de segundas lenguas», Segundas lenguas. Adquisición en el aula. Carmen Muñoz, Ed. (Barcelona: Ariel Lingüística, 2000) 109-125.

19 Heather W. Allen, «Language-Learning Motivation during Short-Term Study Abroad: An Activity Theory Perspective», Foreign Language Annals 43.1 (2010): 27-49.

20 Cita de autores de este artículo.

21 Marc Cadd, «Encouraging Students to Engage with Native Speakers during Study Abroad», Foreign Language Annals 45.2 (2012): 229-245. 
El estudio que presentamos se enmarca, por lo tanto, en una clase pequeña de nivel avanzado, en este contexto híbrido en el que disponemos de instrucción formal al mismo tiempo que los alumnos pueden realizar interacciones naturales que se enmarcan fuera del aula. Aprovechando el contexto concreto en el que los estudiantes estaban estudiando, el tema de las palabras de las que nos ocupamos estaba relacionado con la brujería vasca, un episodio de un tiempo alejado de los alumnos, pero íntimamente asociado a la cultura meta.

\section{Diseño de los test}

Se pidió a los estudiantes que aportaran una lista de palabras nuevas para compartir con sus compañeros en el momento de realizar sus presentaciones (Cf. Metodología, punto 1). Además, debían traer la definición de estas. Como había tres grupos, aportaron tres glosarios que se distribuyeron en clase. Con estas 64 palabras (Cf. Anexo 1) y pertinentes al tema que se trataba en clase, se confeccionaron cuatro test. Siguiendo el modelo usado en Laufer y RozovskiRoitblat, distinguimos entre test de reconocimiento pasivo (passive recognition), es decir, aquel en el que el alumno tiene que elegir el equivalente en inglés de entre cuatro opciones, y un test de memoria pasiva (passive recall), en el cual el estudiante tiene que dar el equivalente en inglés, la L1 de los participantes. A continuación, presentamos un ejemplo de preguntas de cada uno de los dos tipos de test. En el cuadro 1 ilustramos la prueba de reconocimiento pasivo, donde vemos que el estudiante debe elegir entre cuatro posibles traducciones del ítem léxico en cuestión. En el cuadro 2, ilustramos el tipo de pregunta del test de memoria pasiva, en que no se ofrecen opciones para identificar, sino que el alumno tiene que recordar el equivalente en su lengua materna del ítem léxico en cuestión. 


\section{Cuadro 1. Ejemplo de test de reconocimiento pasivo}

Elige de estas cuatro opciones la equivalente en inglés:

1. Abjurar
a) to swear
b) to renounce
c) to suspend
d) to lie

2. Ungido/a(s)
a) ashamed
b) announced
c) anointed
d) angry

3. Supervivencia
a) susceptible
b) custom
c) appearance
d) survival

4. Solar
a) lineage
b) floor
c) farm house
d) whip

5. Sicario
a) daring
b) assassin
c) trail
d) peak

\section{Cuadro 2. Ejemplo de test de memoria pasiva}

Da el equivalente en inglés de las siguientes palabras:

1. Abjurar:

2. Aliado:

3. Aplacar:

4. Aplastante:

5. Arrinconar:

De cada test se realizaron dos versiones con ítems léxicos diferentes para evitar la repetición de palabras (Cf. Anexo 2). Un grupo hizo el test de reconocimiento primero y luego el de memoria y el otro grupo lo hizo en la manera inversa para contrarrestar que uno fuera más sencillo que el otro. Los test se administraron en dos ocasiones: una inmediatamente después de realizar las tareas, el 26 de octubre de 2011, y la segunda ocasión siete semanas después, el 12 de diciembre. De esta forma, siguiendo el diseño del estudio de Laufer y Rozovski-Roitblat ya mencionado, en octubre mediríamos el aprendizaje o la memorización a corto plazo, mientras que en diciembre estaríamos ya hablando de memoria a largo plazo. 


\section{Resultados}

Los resultados de la memoria a corto plazo fueron muy altos, como puede observarse en el cuadro 3. La media de la puntuación de los seis alumnos fue de 28,6 para el test de memoria y de 30,6 para el de reconocimiento sobre un total de 32 puntos. Como era de esperar, el test más exigente, que pedía el equivalente en su L1, arrojó resultados un poco más bajos: dos ítems léxicos menos. En general, podemos concluir que las 64 palabras se retuvieron en un porcentaje muy alto y los alumnos fueron capaces de comprenderlas incluso cuando estas no eran parte de su contexto.

\section{Cuadro 3. Resultados de los test de memoria a corto plazo}

\begin{tabular}{|l|c|c|}
\hline & Test de memoria $(\mathbf{n}=\mathbf{3 2})$ & Test de reconocimiento $(\mathbf{n}=\mathbf{3 2})$ \\
\hline Alumno 1 & 29 & 31 \\
\hline Alumno 2 & 28 & 31 \\
\hline Alumno 3 & 31 & 32 \\
\hline Alumno 4 & 31 & 32 \\
\hline Alumno 5 & 32 & 32 \\
\hline Alumno 6 & 21 & 26 \\
\hline
\end{tabular}

Siete semanas después se volvieron a administrar, durante el tiempo de clase, los mismos test; esta vez con objeto de medir la memoria a largo plazo. No hubo aviso previo de que iba a ser así y los alumnos no habían repasado ni tenido acceso a este vocabulario en los días anteriores al test. Sorprendentemente, las medias fueron muy parecidas a los resultados de los test de memoria a corto plazo. En el caso del test de memoria, la puntuación media del grupo sube a 29,5 y en el caso del test de reconocimiento a 30. Podemos concluir, por lo tanto, que la adquisición de las palabras seleccionadas por medio de las tareas tuvo un efecto duradero. 
Cuadro 4. Resultados de los test de memoria a largo plazo

\begin{tabular}{|l|c|c|}
\hline & Test de memoria $(\mathbf{n}=\mathbf{3 2})$ & Test de reconocimiento $(\mathbf{n}=\mathbf{3 2})$ \\
\hline Alumno 1 & 28 & 30 \\
\hline Alumno 2 & 31 & 30 \\
\hline Alumno 3 & 31 & 30 \\
\hline Alumno 4 & 32 & 32 \\
\hline Alumno 5 & 31 & 32 \\
\hline Alumno 6 & 24 & 26 \\
\hline
\end{tabular}

\section{Comentario de los resultados}

Elaborar tareas de producción que requieran el uso de un vocabulario dado, es un reto para todo profesor, puesto que el alumno siempre puede completar la tarea evitando su uso o por medio de otro vocabulario alternativo. En estas páginas hemos abordado una metodología cuyo objetivo es fijar en la memoria de los alumnos un corpus de palabras relacionadas con el tema que se trabajaba en clase utilizando una metodología por proyectos. Partiendo de varios textos sobre los procesos brujeriles en el País Vasco, los alumnos eligieron y definieron las palabras que necesitaban para resumir un fragmento de dicho texto. Tras una serie de tareas de escritura libre y tres ejercicios de foco en las formas, los alumnos mostraron que eran capaces tanto de reconocer como de proporcionar el equivalente de dicho léxico en inglés. Se concluye que los ítems léxicos quedaron grabados en la memoria a largo plazo porque siete semanas después los alumnos eran capaces de dar el equivalente y elegir correctamente su correspondiente en lengua inglesa.

Coincidimos con Salazar García cuando afirma que «la selección de unidades léxicas destinadas a formar parte de un programa resulta pertinente después de que, en el ámbito específico de la clase, se haya decidido, por parte de los protagonistas del proceso, 
el ámbito nocional $\gg^{22}$. Un léxico encuadrado en tareas encadenadas que los alumnos tienen que seleccionar y manipular se recuerda mejor que listas de vocabulario previamente fijadas en el programa a las que los alumnos tienen que darle la utilidad. En nuestro proyecto, la selección de palabras fue hecha por los propios alumnos a partir de una serie de textos dados. A continuación, tuvieron diferentes oportunidades de usarlas en redacciones en casa y en clase durante un examen. El conocimiento operacional (el saber hacer) se vio manifestado en tareas concretas que los alumnos realizaron y que a su vez sirvió para memorizar el vocabulario propuesto. Como conclusión, podemos afirmar que la metodología adoptada fue adecuada para adquirir un léxico específico pero temáticamente cohesionado.

Una pregunta relacionada con la adquisición de léxico que se plantea es si algunas de las palabras que los alumnos no acertaron al realizar los test fueron cognados ${ }^{23}$. Observando la lista de palabras que fallaron vemos, en primer lugar, que es muy similar en octubre que en diciembre, es decir, que las que no se aprendieron en su momento siguen siendo problemáticas al final del curso. Eso no es sorprendente puesto que no se hizo incidencia pedagógica posterior en este léxico. En cuanto a los cognados de los test de octubre los alumnos fallaron: cátaro (dos veces), peninsular y postulado. En diciembre volvieron a fallar cátaro (una vez), postulado y bula. Si bien estos son cognados en las dos lenguas en cuestión, son conceptos de un ámbito específico de historia, lejos de las experiencias de los alumnos y lejos de la cultura general anglosajona. Por lo tanto, el hecho de ser un cognado no ayuda si el concepto es nuevo para el estudiante, algo que ocurre a menudo con textos históricos y de temas culturales.

Una serie de palabras presentan dificultades añadidas incluso para su comprensión. La palabra entredicho es una de ellas. La mitad de los

22 Víctor Salazar García, «Acercamiento crítico la selección objetiva de contenidos léxicos en la enseñanza de e/le», Estudios de lingüística Universidad de Alicante 18 (2004): 243-273.

23 Rubén Chacón Beltrán, «Towards a Typological Classification of False Cognates EnglishSpanish», Revista Española de Lingüústica Aplicada 19 (2006): 29-39. 
alumnos no fue capaz de dar con su definición, íntimamente ligada a la colocación poner en entredicho. Se trata de un ítem léxico que no puede aprenderse en aislamiento y que necesita de un tratamiento diferente incluso para su comprensión, posiblemente una explicación metalingüística, además del mero significado. Otras piezas léxicas que observamos que también requieren una atención especial a su forma son los siguientes: abjurar, pervivencia, reguero, reticencia y sometimiento. Dos alumnos fallaron estos ítems, lo que nos hace pensar que es necesario algún tratamiento adicional del tipo de foco en las formas, como el que hemos esbozado en el apartado de metodología.

En cualquier caso y a pesar de que hubo palabras que no se retuvieron por medio del trabajo por proyectos, los resultados de este estudio muestran que el $70 \%$ de las nuevas que aparecieron en los textos iniciales fueron retenidas por todos los alumnos del grupo al final del curso. Se trata este de un porcentaje alto que nos anima a seguir utilizando una metodología de proyectos en los cursos de nivel avanzado.

\section{Conclusiones}

Se ha discutido el tipo de actividades que se llevaron a cabo en la clase con objeto de fijar en la memoria una lista de 64 palabras que eran nuevas para los aprendices. La selección y explicación de las piezas léxicas corrió a cargo de los estudiantes, lo cual fue una manera de que empezaran a grabarlas en su memoria. En las producciones posteriores, se vio que los alumnos, tanto en casa con ayuda de notas, diccionarios, etc., como en clase, donde solo contaban con su memoria, hicieron uso de este vocabulario específico. Hubo mucha variación individual en el uso de este léxico en las redacciones escritas en casa, pero curiosamente todos utilizaron más palabras del corpus en la composición del examen en clase que en la realizada en casa, a pesar de que en esta última circunstancia contaban con notas y diccionarios. Los ejercicios de foco en las formas, diseñados para reforzar estas piezas en la memoria, se realizaron siguiendo las 
indicaciones de Laufer y Rozovski-Roitblat, así como los test para medir la adquisición del léxico específico.

En la metodología, se ha tratado de no reducir el tratamiento del vocabulario a la simple traducción de las piezas léxicas, incluyendo tareas de producción libre en las que se vio que los aprendices utilizaron el vocabulario específico. Sin embargo, al medir el conocimiento nos decantamos por la traducción de la pieza léxica en cuestión a la L1 de los alumnos o el reconocimiento de entre varias posibles traducciones. Saber una palabra no es solo entenderla, pero creemos que es un indicador de que esa palabra empieza a fijarse en la memoria, aunque sea de forma pasiva. En futuras investigaciones se podrían diseñar test que incluyan otro tipo de conocimiento, como la categoría léxica, el género u otros aspectos relacionados con saber emplear dichas palabras.

Por tanto, una metodología por proyectos puede y debe reforzarse con ejercicios de escritura dirigidos, así como de tareas de foco en las formas. Hemos descrito cómo se pueden secuenciar dichas actividades, siempre en torno a un tema relevante en el contexto de inmersión en el que los alumnos están viviendo. Una diferencia fundamental que destacan Cenoz y Perales entre un contexto de español como lengua extranjera o un contexto de español como segunda lengua es la posibilidad de visitar de primera mano lugares de la historia local como puede ser una visita a las cuevas de Zugarramurdi (Comunidad Foral de Navarra) o al museo de las brujas que existe en ese mismo enclave, actividades opcionales fuera del horario de clase. Diseñar proyectos como el que hemos mostrado, en el que tratemos de sacar el máximo provecho del contexto de aprendizaje, no solo revierten en la competencia léxica de los estudiantes sino, también, en su comprensión del entorno en el que viven, aunque sea temporalmente. 


\section{Anexo 1}

Lista de vocabulario con su definición y el grupo que lo definió (en negrita los 17 cognados)

1. Abjurar-to renounce, to recant (G2)

2. Akelarre-aquelarre-Witches' sabbath (G3)

3. Aliado/a(s)-allied (G1)

4. Anunciador-advertiser (G3)

5. Aparición-appearance (G1)

6. Aplacar-to soothe $(\mathrm{G} 3)$

7. Aplastante-overwhelming (G2)

8. Arraigar-to become rooted, take root (G3)

9. Arrinconar-to put in a corner (G2)

10. Auge-peak (G3)

11. Azote-whip, lash (G1)

12. Baskonia-Basque Country (G2)

13. Brujeril-related to witches (G1)

14. Bula-bull (G1)

15. Capellán-chaplain (G2)

16. Caserío-farm house (G3)

17. Cátaro-Cathar (G1)

18. Caza (de brujas)-(witch) hunt (G1) (G3)

19. Comadrona-midwife (G1)

20. Comisario-commissioner (G1)

21. Condenar-to condemn (G1)

22. Cucurucho-cone (G1)

23. Chivato-informer, stool pigeon (G3)

24. Desatar-to untie-to undo (G2)

25. Desenmascarar-to unmask (G2)

26. Dominico, ca-Dominican(G1)

27. Eclesial-ecclesiastical(G1)

28. Entredicho-to be in doubt (G3)

29. Ermita-chapel (G3)

30. Escaso/a(s)-scant (G3)

31. Espada-sword (G3) 
32. Excomulgar-to excommunicate (G2) (G3)

33. Franciscano-Franciscan (G1)

34. Galera-galley (G1)

35. Hechizo-spell (G1)

36. Herejía-heresy (G2)

37. Hoguera-bonfire (G1)

38. Indefensión-defenselessness (G3)

39. Macho cabrío-billy goat (G3)

40. Monigote-rag doll (G1)

41. Natalidad-birthrate (G3)

42. Osadía-daring, boldne (G1)ss

43. Peninsular-peninsular (G1)

44. Pervivencia-survival (G3)

45. Pirenaico/a(s)-from the Pyrenees (G2)

46. Pista-trace (G2)

47. Plegar-to fold (G3)

48. Postulado-postulate (G3)

49. Prado-field (G3)

50. Promulgar-to enact (G1)

51. Rama-branch $(\mathrm{G} 2)$

52. Reguero-trail (G2)

53. Resaltar-to stand out (G2)

54. Reticencia-reluctance (G1)

55. Sacerdotisa-priestess (G3)

56. Sede-seat, see (G1)

57. Sicario-hired assessin (G2)

58. Solar-lineage (G3)

59. Sometimiento-subjection (G1)

60. Supervivencia-survival (G3)

61. Terrenal-worldly,earthly (G3)

62. Ungido/a(s)-anointed (G2)

63. Vidente-clairvoyance $(\mathrm{G} 2)$

64. Vislumbrar-to make out, to discern (G2) 


\section{Anexo 2}

\section{Test utilizados para comprobar la adquisición del léxico}

\section{test de memoria versión 1}

Da el equivalente en inglés de las siguientes palabras:

1. Abjurar

2. Aliado

3. Aplacar

4. Aplastante

5. Arrinconar

6. Auge

7. Baskonia

8. Cátaros

9. Caza

10. Comadrona

11. Comisario

12. Condenar

13. Cucurucho

14. Chivato

15. Desatar

16. Entredicho

17. Escaso/a(s)

18. Franciscano

19. Galera

20. Hechizo

21. Hoguera

22. Indefensión

23. Monigote

24. Natalidad

25. Peninsular 
26. Pervivencia

27. Plegar

28. Rama

29. Sicario

30. Solar

31. Supervivencia

32. Ungido/a(s)

\section{test de memoria versión 2}

Da el equivalente en inglés de las siguientes palabras:

1. Akelarre

2. Anunciador

3. Aparición

4. Arraigar

5. Azote

6. Brujeril

7. Bula

8. Capellán

9. Caserío

10. Desenmascarar

11. Dominico

12. Eclesial

13. Ermita

14. Espada

15. Excomulgar

16. Herejía

17. Macho cabrío

18. Osadía

19. Pirenaico

20. Pista

21. Postulado 
22. Prado

23. Promulgar

24. Reguero

25. Resaltar

26. Reticencia

27. Sacerdotisa

28. Sede

29. Sometimiento

30. Terrenal

31. Vidente

32. Vislumbrar

\section{test de reconocimiento versión 1}

Elige de estas cuatro opciones la equivalente en inglés:

1. Abjurar
a) to swear
b) to renounce
c) to suspend
d) to lie

2. Ungido/a(s)
a) ashamed
b) announced
c) anointed
d) angry

3. Supervivencia
a) susceptible
b) custom
c) appearance
d) survival

4. Solar
a) lineage
b) floor
c) farm house
d) whip

5. Sicario
e) daring
b) assassin
c) trail
d) peak

6. Monigote
a) cone
b) chapel
c) monkey
d) rag doll

7. Natalidad
a) birthrate
b) fertility
c) demography d) childhood

8. Peninsular
a) relative to Europe
b) relative to Spain
c) relative to the islands
d) relative to the Peninsula 
9. Pervivencia
a) defense
b) survival
c) defiance
d) defeat

10. Plegar
a) to fold
b) to cry
c) to fall
d) to decay

11. Rama
a) $\log$
b) root
c) wood
d) branch

12. Indefensión
a) ignorance
b) defenselessness
c) timelessness d) destiny

13. Hoguera
a) blindness
b) firework
c) bonfire
d) hunt

14. Hechizo
a) spell
b) blessing
c) punishment
d) torture

15. Galera
a) navy
b) storm
c) heresy
d) galley

16. Aliado
a) allied
b) aligned
c) alleged
d) alien

17. Aplacar
a) to fight
b) to conquer
c) to win
d) to soothe

18. Aplastante
a) overwhelming
b) intriguing c) overdone
d) overcautious

19. Arrinconar
a) to condemn
b) to untie
c) to put in a corner
d) to lie

20. Auge
a) future
b) rise
c) autonomy
d) peak

21. Baskonia
a) Basque Country
b) Basque farm
c) Basque language
d) Basque goddess

22. Cátaro
a) punishment
b) cathar
c) goat
d) caucasus

23. Caza
a) chapel
b) cone
c) hunt
d) rag doll

24. Chivato
a) informer
b) billy goat
c) sword
d) daring

25. Comadrona
a) birthrate
b) witch
c) survival
d) midwife 
26. Franciscano
a) painting
b) Franciscan
c) church
d) French

27. Escaso/a(s)
a) scant
b) chapel
c) advertiser
d) heresy

28. Entredicho
a) repeated
b) overheared
c) enacted
d) in doubt

29. Desatar
a) to untie
b) to soothe
c) to defy
d) to unravel

30. Cucurucho
a) conch
b) cooper
c) cone
d) branch

31. Condenar
a) condole
b) conduct
c) condense
d) condemn

32. Comisario
a) commander
b) commissioner
c) commercial
d) compassionate

\section{test de reconocimiento versión 2}

Elige de estas cuatro opciones la equivalente en inglés:

1. Vislumbrar
a) to discern
b) to forgive
c) to review
d) to retell

2) Vidente
a) public
b) clairvoyance
) invisible
d) blind

3) Terrenal
a) ground
b) soothing
c) tender
d) earthly

4) Sometimiento
a) reluctance
b) subjection
c) enactment
d) appearance

5) Promulgar
a) to inform
b) to enact
c) to promise
d) to condemn

6) Sede
a) seat
b) thirst
c) amount of time d) trace

7) Sacerdotisa
a) healer
b) witch
c) lover
d) priestess

8) Reticencia
a) daring
b) cone
c) reluctance
d) jealousy

9) Resaltar
a) stand out
b) to steal
c) to attack
d) to condemn 
10) Reguero

11) Prado
a) bonfire
b) trail
c) garden
d) branch
a) trail
b) seat
c) painting
d) field

12) Postulado
a) posting
b) postpone
c) postulate
d) postergate

13) Pista
a) trace
b) branch
c) field
d) chapel

14) Pirenaico/a(s)
a) relative to the Pyrenees
b) relative to the Pireo
c) relative to the mountain
d) relative to the pyramids

15) Osadía
a) priest
b) daring
c) big bear
d) lineage

16) Macho cabrío
a) male chauvinist b) withes sabbath
c) billy goat
d) sheep

17) Herejía
a) farm house
b) heresy
c) clairvoyance
d) peak

18) Excomulgar
a) to communicate
b) to excommunicate
c) to exchange
d) to expand

19) Espada
a) sword
b) chapel
c) branch
d) helmet

20) Ermita
a) tree
b) poison
c) chapel
d) witch

21) Eclesial
a) relative to the church
b) relative to the baptism
c) relative to the communion
d) relative to confession

22) Dominico
a) dominical
b) Sunday
c) Dominican
c) domino

23) Desenmascarar
a) to hunt
b) to lie
c) to whip
d) to unmask

24) Caserío
a) cathar
b) farm house
c) farmer
d) piece of land

25) Capellán
a) chaplain
b) song
c) trace
d) advertiser

26) Bula
a) peak
b) mountain
c) bull
d) spell

27) Brujeril
a) related to witchesb) hunting
c) midwife
d) related to spells 
28) Azote
a) bonfire
b) whip
c) allied
d) cave

29) Arraigar
a) to take over
b) to leave
c) to take root
d) to reveal

30) Aquelarre
a) dance
b) villeage
c) spell
d) witches' sabbath

31) Aparición
a) appearance
b) punishment
c) reluctance
d) overwhelming

32) Anunciador
a) advertiser
b) peninsular
c) daring
d) put in a corner 